Taeho Woo

\title{
Atomic Information Technology
}

Safety and Economy of Nuclear Power Plants

Dpringer 


\section{Contents}

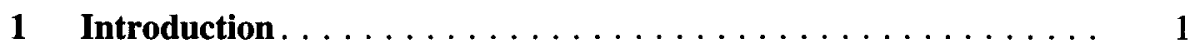

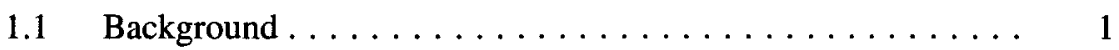

1.2 Basic Statistics. . . . . . . . . . . . . . . . . 3

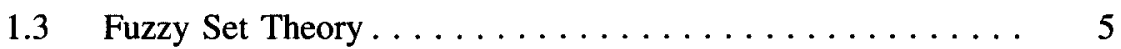

1.4 Artificial Neural Network $\ldots \ldots \ldots \ldots \ldots \ldots \ldots \ldots \ldots \ldots$

1.5 System Dynamics. . . . . . . . . . . . . . . . . 6

References ......................... 8

2 Dynamical Modeling of Economy in Global Nuclear

Energy Market. . . . . . . . . . . . . . . . . . . . 11

2.1 Introduction $\ldots \ldots \ldots \ldots \ldots \ldots \ldots \ldots \ldots \ldots \ldots \ldots$

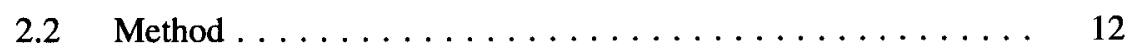

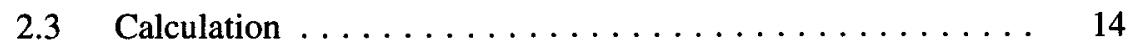

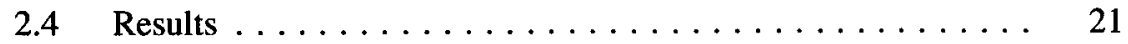

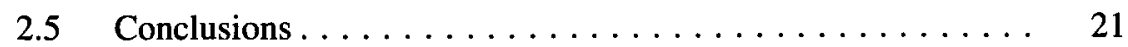

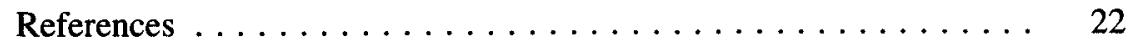

3 Assessment of National Nuclear Fuel Cycle for Transmutations

of High Level Nuclear Waste $\ldots \ldots \ldots \ldots \ldots \ldots \ldots \ldots \ldots \ldots$

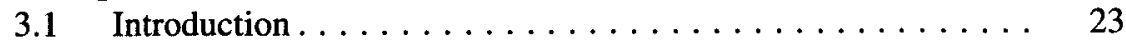

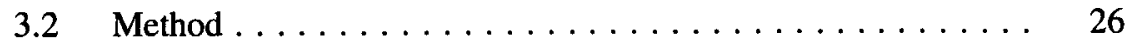

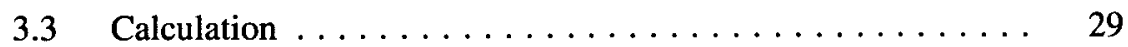

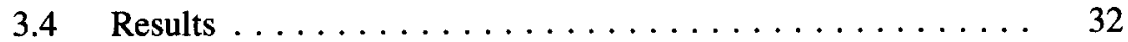

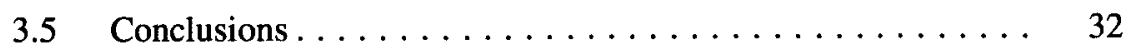

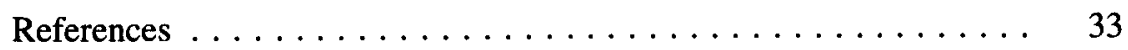

4 Dynamical Management of Atomic-Multinology in the Aspect

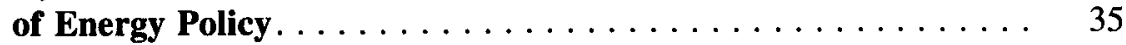

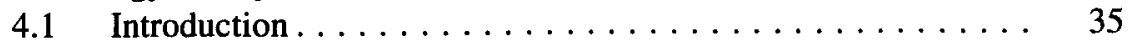

4.2 Method ....................... 39 


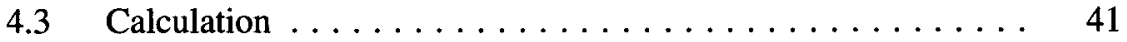

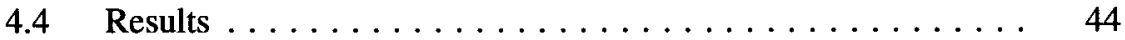

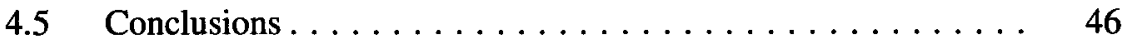

References ...................... 47

5 Technological Management of Atomic-Multinology

by Social Network Theory . . . . . . . . . . . . . . . . . . 49

5.1 Introduction . . . . . . . . . . . . . . . . 49

$5.2 \quad$ Method ............................ 53

5.3 Calculation ...................... 54

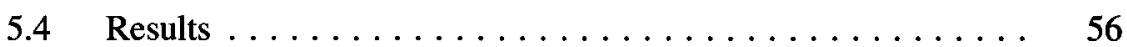

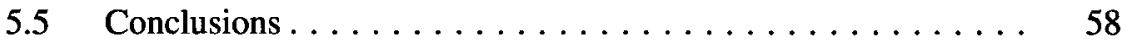

References ........................ 59

6 Nuclear Safety Assessment for the Passive System of the Nuclear Power Plants (NPPs) in Safety Margin Estimation. . . . . . . . . 61

$6.1 \quad$ Introduction . . . . . . . . . . . . . . . 62

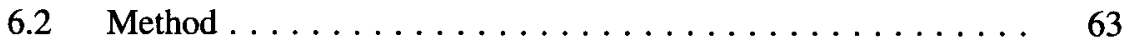

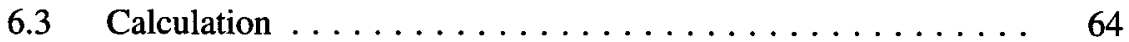

6.4 Results and Discussions. . . . . . . . . . . . . 66

6.5 Conclusions . . . . . . . . . . . . . . . . . . . 69

References ....................... 72

7 Non-linear Dynamical Reliability Analysis in the Very High

Temperature Gas Cooled Reactor . . . . . . . . . . . . . . 75

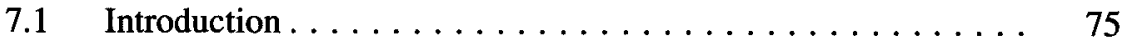

7.2 Dynamics of the Resistance-Stress Method . . . . . . . . . 76

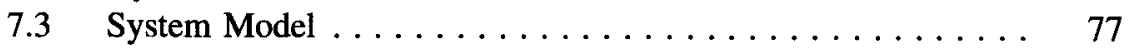

$7.4 \quad$ Calculation $\ldots \ldots \ldots \ldots \ldots \ldots \ldots \ldots \ldots \ldots \ldots \ldots$

$7.5 \quad$ Results and Discussions. . . . . . . . . . . . . 82

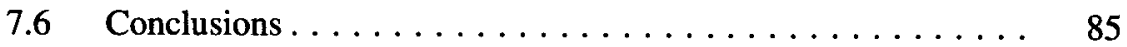

References ...................... 88

8 Dynamical Seismic Probabilistic Safety Assessment

for Earthquake. . . . . . . . . . . . . . . . . 89

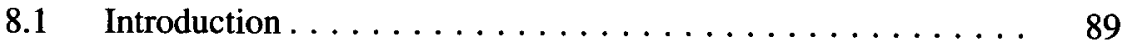

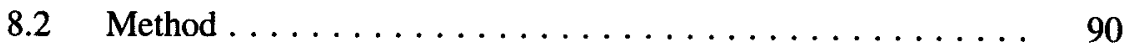

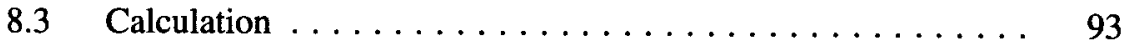

$8.4 \quad$ Results $\ldots \ldots \ldots \ldots \ldots \ldots \ldots \ldots \ldots \ldots \ldots \ldots \ldots \ldots \ldots$

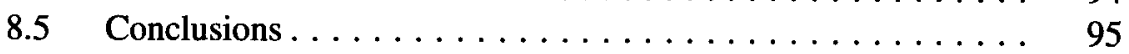

References ...................... 97 
9 Safety Assessment for Lunar Nuclear Power Reactor

in Cooling Accident $\ldots \ldots \ldots \ldots \ldots \ldots \ldots \ldots \ldots \ldots$

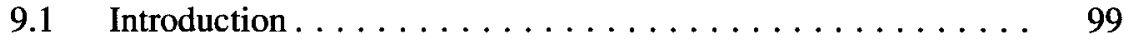

9.2 Method . . . . . . . . . . . . . . . . . . . . 104

9.3 Calculation . . . . . . . . . . . . . . . . 106

$9.4 \quad$ Results . . . . . . . . . . . . . . . . . . . . . . . . . . . . 109

9.5 Conclusions . . . . . . . . . . . . . . . . . . 111

References . . . . . . . . . . . . . . . . . 116

10 Nuclear Safeguard Management of Operation Security

in Nuclear Power Plants . . . . . . . . . . . . . . . . . . . . . . . . . . . 119

10.1 Introduction . . . . . . . . . . . . . . . . . . . . . . . 119

10.2 Factors for Modeling $\ldots \ldots \ldots \ldots \ldots \ldots \ldots \ldots \ldots \ldots \ldots \ldots$

10.2.1 Safeguard Measures Factor . . . . . . . . . 121

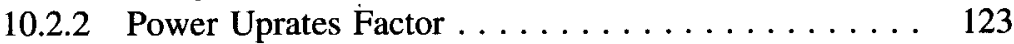

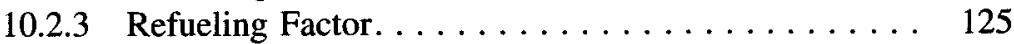

10.3 Dynamical Method . . . . . . . . . . . . . 126

10.3.1 Network Effect. . . . . . . . . . . . . 126

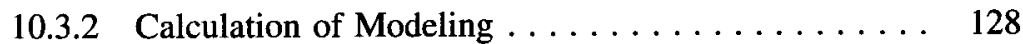

10.4 Results ........................... 131

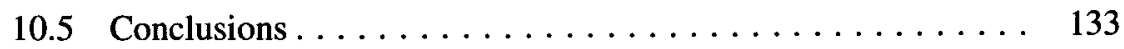

References .......................... 134

11 Life Extension Assessment for Safeguard in Nuclear

Power Plants (NPPs) Using a Production Function . . . . . . . 135

11.1 Introduction . . . . . . . . . . . . . . . . . 135

11.2 Nuclear Safeguard Estimator Function for Modeling. . . . . . 139

11.3 Dynamical Method . . . . . . . . . . . . . . 141

11.4 Technology Assessment . . . . . . . . . . . . . 142

11.5 Results . . . . . . . . . . . . . . . . . . 142

11.6 Conclusions . . . . . . . . . . . . . . . . . 143

References ......................... 147

12 Security Investigations in Nuclear Materials Using

Analytic Pair Values. . . . . . . . . . . . . . . . . . . . . 149

12.1 Introduction . . . . . . . . . . . . . . . . . 149

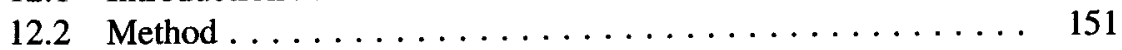

12.3 Results ......................... 155

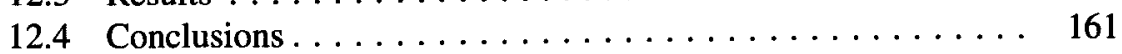

References ........................ 162

13 Nuclear Security Assessment Using Loss Function with Modified Random Numbers. . . . . . . . . . . . . . 165

13.1 Introduction . . . . . . . . . . . . . . 165 
13.2 Method $\ldots \ldots \ldots \ldots \ldots \ldots \ldots \ldots \ldots \ldots \ldots \ldots \ldots \ldots \ldots \ldots$

13.2.1 Game Theory.................. 167

13.2.2 Safeguard Factor $\ldots \ldots \ldots \ldots \ldots \ldots \ldots \ldots \ldots, 171$

13.2.3 Dynamical Method.................. 172

13.3 Results . . . . . . . . . . . . . . . . . . 173

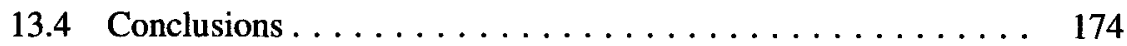

References ....................... 178

14 Conclusion $\ldots \ldots \ldots \ldots \ldots \ldots \ldots \ldots \ldots \ldots \ldots \ldots \ldots \ldots \ldots$ 